\title{
The relationship between synapsis, recombination nodules and chiasmata in tomato translocation heterozygotes
}

\author{
LISA HERICKHOFF*, STEPHEN STACK* $\dagger$ \& JAMIE SHERMAN*† \\ *Department of Biology and $\dagger$ Cell and Molecular Biology Program, Colorado State University, Fort Collins, Colorado \\ 80523, U.S.A.
}

\begin{abstract}
Spreads of synaptonemal complexes (SCs) were made from five different reciprocal translocation heterozygotes in tomato (Lycopersicon esculentum). Patterns of synapsis and distribution of recombination nodules (RNs) and chiasmata were analysed. Translocated chromosomes usually synapsed to form quadrivalents. By measuring the length of synapsed arms, we estimated the positions of the breaks for translocations $2-10 \mathrm{~b}$ (both breaks in euchromatin), $9-12$ (both breaks in heterochromatin), and 2-10a, 1-3 and 1-12 (one break in euchromatin and one break in heterochromatin). The distribution of RNs indicates that interference occurs within arms of quadrivalents. If interference occurs between arms it is transferred to opposite and adjacent arms equally. We found no significant difference between the distribution of RNs and chiasmata. These results support a one RN: one crossover: one chiasma relationship.
\end{abstract}

Keywords: chiasma, interference, recombination nodules, synapsis, synaptonemal complex, translocation heterozygote.

\section{Introduction}

In some plants there appears to be a $1: 1$ relationship between crossing over and homologous synapsis, i.e., synaptonemal complex (SC) formation. For example, Rhoeo spathacea is a complete translocation heterozygote in which the frequency of synapsed chromosome ends is the same as the frequency of ends held together by chiasmata (Stack \& Soulliere, 1984). Because homologously synapsed ends are separated by asynapsed segments, perhaps asynapsis interrupts transmission of crossover interference and assures that these isolated synapsed segments are free to crossover. Alternatively, since the synapsed ends are separated by non-synapsed segments that comprise approximately 70 per cent of the length of each bivalent, perhaps interference decreases to the point of being ineffective.

Tomato translocation heterozygotes have a number of advantages for testing these hypotheses [as well as other relationships such as that of recombination nodules (RNs) to chiasmata]:

1 a variety of different translocations are available in tomato (Rick, 1985);

Correspondence: Lisa Herickhoff, Department of Botany, Arizona State University, Tempe, AZ 85287, U.S.A.
2 tomato has a diploid complement of 24 small chromosomes with relatively large blocks of pericentric heterochromatin (Stack \& Anderson, 1986a). By selecting translocations having breaks in either euchromatin or heterochromatin or both, possible effects of the break site on synapsis and crossing over can be examined;

3 if translocated chromosomes synapse homologously, they will form cross-shaped quadrivalents with four segments of synapsed SC separated by asynapsed lateral elements at the region of partner exchange. The presence or absence of crossing over within the quadrivalent will lead to one of six distinct configurations at diakinesis: four univalents (no chiasmata), a bivalent and two univalents (one or more chiasmata in one arm only), two bivalents (one or more chiasmata in two non-adjacent arms only), a trivalent and a univalent (one or more chiasmata in two adjacent arms only), a chain of four (one or more chiasmata in three arms only), and a ring of four (one or more chiasmata in all four arms);

4 based on tomato's linkage map length of 1063 cM (Tanksley \& Mutschler, 1990), there should be about $(1063 \mathrm{cM} \div 50 \mathrm{cM} /$ crossover $=) 21$ crossovers scattered among twelve bivalents. With 23 euchromatic arms (chromosome 2 has a heterochromatic short arm) 
there will be slightly fewer than an average of one crossover per arm, thus it cannot be assumed that every arm of a quadrivalent will have a crossover. As a result, many or all of the six distinct diakinesis configurations should be observed;

5 based on tomato's linkage map, the average number of crossovers is similar to the average number of RNs observed at mid- to late pachytene, as is expected if there is a 1:1 relationship between crossovers and RNs (Stack \& Anderson 1986a,b; Sherman et al., 1992). This suggests that we are missing few, if any, RNs due to experimental technique (Sherman et al., 1992) or ephemeral RNs (see von Wettstein et al., 1984, for a review);

6 the small numbers of RNs and chiasmata allow one to perform an independent test of the one $\mathrm{RN}$ : one crossover (chiasma) relationship in tomato. Thus if RNs are indicative of crossing over and chiasmata, then the distribution of RNs among the SCs associated with translocated chromosomes should predict the distribution of chiasmata between translocated chromosomes at diakinesis;

7 because each of tomato's 12 SCs can be identified on the basis of its relative length, arm ratio, and fraction of each arm that is heterochromatic (Sherman \& Stack, 1992), the distribution of RNs can be determined for each chromosome.

In our study we investigated five different reciprocal translocation heterozygotes chosen to compare effects of paired breaks in euchromatin and heterochromatin (2-10a, 1-12, 1-3), euchromatin only $(2-10 \mathrm{~b})$, and heterochromatin only (9-12). For the translocated chromosomes we determined the patterns of synapsis, distributions of RNs during pachytene and distributions of chiasmata during diakinesis. By analysing and comparing these data we have: (i) estimated the positions of all the translocation breaks; (ii) determined that location of breaks in euchromatin vs. heterochromatin does not affect the extent of synapsis; (iii) observed that not all synapsed arms of quadrivalents have RNs or form chiasmata; (iv) determined that RNs show interference within arms of quadrivalents; ( $v$ ) showed that crossing over is generally depressed among translocated chromosomes; and (vi) demonstrated a significant correlation between RNs and chiasmata for each translocation heterozygote, thus supporting the $1 \mathrm{RN}$ : 1 crossover: 1 chiasma relationship in tomato.

\section{Materials and methods}

Tomato seeds that were homozygous for one of five different translocations (Table 1) were germinated, and the seedlings were grown to maturity in a greenhouse maintained at 20 to $25^{\circ} \mathrm{C}$. These plants were crossed with normal diploid tomato plants (var. cherry) by separating the calyx and corolla of buds approximately $7 \mathrm{~mm}$ in length, removing each immature stamen, and then placing a small amount of mature pollen from a normal tomato plant on the stigma of the emasculated flower. Each bud was covered with a small rectangular envelope that was slightly larger than the bud. The envelopes were then taped shut. Envelopes were removed when visible fruits were present. Fruits were allowed to ripen and $F_{1}$ seeds harvested. Seeds were dried and stored at $4{ }^{\circ} \mathrm{C}$.

$\mathrm{F}_{1}$ seeds were planted and grown to maturity. These heterozygous plants were maintained by asexual propagation. Anthers used for experimentation were harvested from plants that were 2-3 months old.

Spreads of SCs were prepared, fixed, silver stained, and picked up on grids as described by Sherman et al. (1992).

Sets of SCs were examined and photographed at a magnification of $\times 6000$ using an AEI 801 electron microscope. Magnifications were determined using a carbon replica grating. SCs on photographic prints were traced onto acetate sheets. Heterochromatin, RNs, telomeres, and kinetochores were marked. Tracings were then analysed using a Hewlett Packard 86B computer, a HP 9111A graphics tablet, and a program specifically written for this purpose.

SCs were staged according to Stack \& Anderson (1986a,b), using the following criteria. In late zygotene, synapsis is not complete, and kinetochores do not stain. In early pachytene, synapsis is complete, and kinetochores are not stained. However, the position of kinetochores is indicated by segments of thickened lateral elements. The thinner lateral elements to either side indicate the location of pericentric heterochro-

Table 1 Translocations in tomato. Stocks are available from C. M. Rick, Vegetable Crops Department, University of California, Davis, California 95616. Taken from Gill, et al. (1980)

\begin{tabular}{|c|c|c|c|c|}
\hline Translocation & $\begin{array}{l}\text { Davis LA } \\
\text { number }\end{array}$ & Source & Variety & Treatment \\
\hline $1-3$ or 8 & 1905 & $\begin{array}{l}\text { Stringham } \\
51-9 b\end{array}$ & $\begin{array}{l}\text { Early Fire } \\
\text { Ball }\end{array}$ & $\begin{array}{l}\text { Thermal } \\
\text { neutrons }\end{array}$ \\
\hline $1-12$ & 1896 & $\begin{array}{l}\text { Stringham } \\
47-27 b\end{array}$ & $\begin{array}{l}\text { Early Fire } \\
\text { Ball }\end{array}$ & $\begin{array}{l}\text { Ethylene } \\
\text { oxide }\end{array}$ \\
\hline $2-10 a$ & 1898 & $\begin{array}{l}\text { Stringham } \\
48-2 \mathrm{a}\end{array}$ & $\begin{array}{l}\text { Early Fire } \\
\text { Ball }\end{array}$ & $\begin{array}{l}\text { Ethylene } \\
\text { oxide }\end{array}$ \\
\hline $2-10 b$ & 1906 & $\begin{array}{l}\text { Stringham } \\
51-11 \mathrm{a}\end{array}$ & $\begin{array}{l}\text { Early Fire } \\
\text { Ball }\end{array}$ & $\begin{array}{l}\text { Thermal } \\
\text { neutrons }\end{array}$ \\
\hline $9-12$ & $\begin{array}{l}1115 \\
1890\end{array}$ & $\begin{array}{l}\text { Burdick } \\
3-8\end{array}$ & $\begin{array}{l}\text { Hybrid } \\
\text { backgrounc }\end{array}$ & Unknown? \\
\hline
\end{tabular}


matin. Lateral elements in distal euchromatin are, again, thicker than in heterochromatin. In middle pachytene, synapsis is complete, and kinetochores stain lightly at sites of thickened lateral elements in pericentric heterochromatin. In late pachytene, synapsis is complete, and kinetochores stain darkly. In early diplotene, desynapsis has begun, and kinetochores stain darkly. RN data were collected only from SCs in midto late pachytene.

To obtain data on chiasmata, anthers from translocation heterozygotes containing primary microsporocytes at diakinesis were fixed in 1:3 acetic ethanol for at least one hour before squashing in 45 per cent acetic acid. Slides were stained with aceto-orcein, and cover glasses were mounted in Euparal. Squashes were examined and photographed by bright field light microscopy using a green filter.

Statistical analyses were performed using an IBM compatible computer with the program GraphPAD InStat version 1.12a (GraphPAD software). For a comparison of the number of RNs in normal versus translocated chromosomes, an unpaired, two-tailed $t$-test was performed. Chi-squared analyses were used to compare the frequencies of chiasma and RN configurations.

\section{Results}

\section{Locating translocation breakpoints by patterns of synapsis}

In nuclei from translocation heterozygotes 1-3, 1-12, $2-10 \mathrm{a}$, and $9-12$, the most common pattern of synapsis of the translocated chromosomes was a quadrivalent in the shape of a four-armed cross with some asynapsis in areas of partner exchange (Figs 1-3). Chromosomes involved in translocation 2-10b commonly synapsed as a cross with only three arms due to the near terminal location of the break on chromosome 10 (Fig. 4). In all nuclei without a quadrivalent, the translocated chromosomes synapsed as bivalents where one arm must be at least partially synapsed non-homologously.

While there is some variation in the length of SCs from spread to spread, relative lengths of chromosomes within a spread are consistent (Sherman \& Stack, 1992). Therefore to compare translocated SCs in different spreads, measurements of synapsed and asynapsed lateral elements were made in micrometres and then converted to percentages of the total length of the lateral element. The midpoints of asynapsed areas were taken as apparent sites of translocation break points (Moses et al., 1977; Ashley \& Russell, 1986). For each translocation heterozygote these sites were expressed as a percentage of the length of the arm measured from the centromere. The most probable position for each break was estimated by averaging midpoints of asynapsed areas (Fig. 5).

Translocation 1-3. The translocated chromosomes synapsed as quadrivalents in cross configurations in 85 per cent of the 26 nuclei $(22 / 26)$ analysed (Fig. 1). Among quadrivalents, non-translocated chromosome 1 averaged 89.5 per cent $( \pm 10.3$ per cent) of its length synapsed, while non-translocated chromosome 3 averaged 83.6 per cent $( \pm 13.1$ per cent $)$ of its length synapsed. The break in chromosome 1 appears to be in heterochromatin 7.0 per cent $( \pm 1.1$ per cent) of the length of the long arm from the centromere. The break in chromosome 3 appears to be located in euchromatin at a point 43.5 per cent $( \pm 4.9$ per cent $)$ of the length of the long arm from the centromere (Fig. 5).

Translocation 1-12. The translocated chromosomes synapsed as quadrivalents in cross configurations in 86 per cent of the 21 nuclei $(18 / 21)$ analysed. Among quadrivalents, non-translocated chromosome 1 averaged 86.9 per cent $( \pm 9.5$ per cent $)$ of its length synapsed, while non-translocated chromosome 12 averaged 83.7 per cent $( \pm 7.1$ per cent $)$ of its length synapsed. The break in chromosome 1 appears to be in euchromatin 43.6 per cent $( \pm 10.1$ per cent) of the length of the long arm from the centromere. The break in chromosome 12 appears to be in heterochromatin 10.4 per cent $( \pm 1.1$ per cent) of the length of the long arm from the centromere (Fig. 5).

Translocation 2-10a. The translocated chromosomes synapsed as quadrivalents in cross configurations in 85 per cent of the 20 nuclei $(17 / 20)$ analysed (Fig. 2). Among quadrivalents, non-translocated chromosome 2 averaged 89.9 per cent $( \pm 6.5$ per cent) of its length synapsed, while non-translocated chromosome 10 averaged 84.1 per cent $( \pm 8.7$ per cent) of its length synapsed. The break in chromosome 2 appears to be in euchromatin 32.6 per cent $( \pm 4.5$ per cent) of the length of the long arm from the centromere. The break in chromosome 10 appears to be located in heterochromatin at a point 7.9 per cent ( \pm 1.1 per cent) of the length of the long arm from the centromere (Fig. 5).

Translocation 2-10b. The translocated chromosomes synapsed as quadrivalents in a three-armed cross configuration or a four-armed cross with one minute arm in 92 per cent of the 25 nuclei $(23 / 25)$ analysed (Fig. 4). Among quadrivalents, non-translocated chromosome 2 averaged 87.8 per cent $( \pm 4.8$ per cent) of its length synapsed, while non-translocated chromo- 

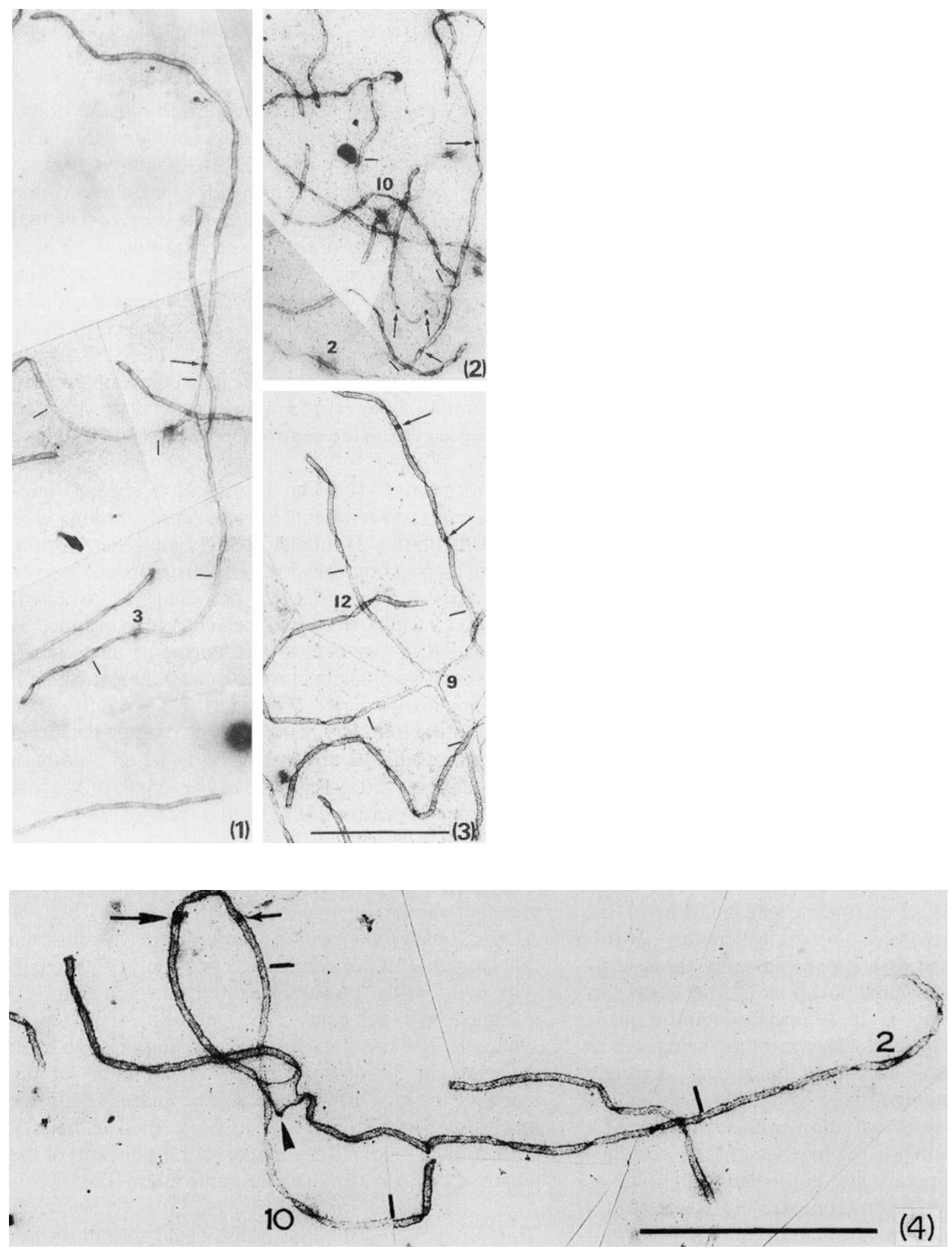

Figs 1-4 Montages of translocation heterozygote quadrivalents at pachytene. Small arrows point to RNs, lines indicate heterochromatin/euchromatin borders. Numbers near centromeres indicate chromosome numbers. 1 Translocation 1-3. 2 Translocation 2-10a. 3 Translocation 9-12. 4 Translocation 2-10b (arrowhead indicates the minute fourth arm of 2-10b. Stain precipitate is visible on the SC in the upper left [large arrow]). Bar in Fig. 3 for Figs 1-3 and bar in Fig. 4 represent $5 \mu \mathrm{m}$. 
Fig. 5 Breakpoints in pachytene chromosomes involved in reciprocal translocations $1-3,1-12,2-10 \mathrm{a}$, 2-10b, and 9-12. The number of each translocation is indicated to the left with the two chromosomes involved in the translocation diagrammed immediately below. Each chromosome's number is indicated to the right. Chromosomes are illustrated according to Sherman \& Stack (1992) where thick lines represent euchromatin, thin lines represent pericentric heterochromatin, and circles represent kinetochores. The most probable location of the break is indicated by the arrow heads with the lines to either side representing one standard deviation. The bar represents $10 \mu \mathrm{m}$.


2

10

2

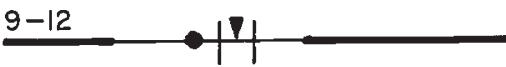

12 some 10 averaged 89.9 per cent ( \pm 6.2 per cent) of its length synapsed. The break in chromosome 2 appears to be in euchromatin 72.1 per cent $( \pm 9.1$ per cent $)$ of the length of the long arm from the centromere. The break in chromosome 10 appears to be located in euchromatin at a point 89.7 per cent $( \pm 1.8$ per cent $)$ of the length of the long arm from the centromere (Fig. 5).

Translocation 9-12. The translocated chromosomes synapsed as quadrivalents in cross configurations in 85 per cent of the 28 nuclei (24/28) analysed (Fig. 3). Among quadrivalents, non-translocated chromosome 9 averaged 88.5 per cent $( \pm 10.2$ per cent $)$ of its length synapsed, while non-translocated chromosome 12 averaged 85.6 per cent ( \pm 10.5 per cent) of its length synapsed. The break in chromosome 9 appears to be in heterochromatin at a point 7.0 per cent ( \pm 1.0 per cent) of the length of the long arm from the centromere. The break in chromosome 12 appears to be located in heterochromatin at a point 16.6 per cent $( \pm 1.5$ per cent) of the length of the long arm from its centromere (Fig. 5).

\section{Effects of translocations on frequency of recombination nodules}

Frequencies of RNs associated with translocated chromosomes synapsed as quadrivalents were compared with frequencies of RNs associated with the same non-translocated chromosomes homologously synapsed in bivalents. The latter data was reported by Sherman et al. (1992).

Chromosome 1. For translocation 1-3, there was an average of 1.4 ( $\pm 0.4, n=22$ quadrivalents observed) RNs associated with chromosome 1 . For translocation $1-12$, there was an average of $1.6( \pm 0.5, n=18$ quadrivalents observed) RNs associated with chromosome 1. The averages for the two translocated chromosomes are not significantly different from each other $(P=0.1732)$. Both of these averages are significantly less than the average of 2.4 ( $\pm 0.5, n=43$ SCs observed) RNs observed on normal chromosome 1 bivalents $(P<0.0001)$.

Chromosome 2. For translocation 2-10a, there was an average of 1.8 ( $\pm 0.6, n=17$ quadrivalents observed) RNs associated with chromosome 2 . In translocation $2-10 \mathrm{~b}$, there was an average of $1.8( \pm 0.4, n=23$ quadrivalents observed) RNs associated with chromosome 2 . These averages are the same and not significantly different from the average of $1.9( \pm 0.6, n=43$ SCs observed) RNs observed on the long arm of normal chromosome 2 bivalents $(P=1.0,0.4$ and 0.38 , respectively).

Chromosome 3. For translocation 1-3, there was an average of 1.2 ( $\pm 0.5, n=22$ quadrivalents observed) RNs associated with chromosome 3 . This is significantly less than the average of $2.0( \pm 0.5, n=43$ SCs 
observed) RNs observed on normal chromosome 3 bivalents $(P<0.0001)$.

Chromosome 9. For translocation 9-12, there was an average of 1.2 ( $\pm 0.5, n=24$ quadrivalents observed) $\mathrm{RNs}$ associated with chromosome 9 . This average is not significantly less than the average of $1.5( \pm 0.6$, $n=46$ SCs observed) RNs observed on normal chromosome 9 bivalents $(P=0.062)$.

Chromosome 10. For translocation 2-10a, there was an average of $1.1( \pm 0.4, n=17$ quadrivalents observed) RNs associated with chromosome 10 . For translocation $2-10 \mathrm{~b}$, there was an average of 1.3 ( $\pm 0.6, n=23$ quadrivalents observed) RNs associated with chromosome 10 . These averages are not significantly different from one another $(P=0.24)$ but significantly less than the average of $1.6( \pm 0.5, n=44$ SCs observed) RNs observed on normal chromosome 10 bivalents ( $P=0.003$ and $P=0.0514$, respectively).

Chromosome 12. For translocation 1-12, there was an average of 0.65 ( $\pm 0.4, n=22$ quadrivalents observed) $\mathrm{RNs}$ associated with chromosome 12 . For translocation 9-12, there was an average of $1.08( \pm 0.6, n=24$ quadrivalents observed) RNs associated with chromo- some 12 . These averages are significantly different from one another $(P<0.0001)$, and both are significantly less than the average of $1.5( \pm 0.5, n=38$ SCs observed) RNs observed on normal chromosome 12 bivalents $(P<0.0001, P=0.0015$, respectively).

\section{Recombination nodule distribution within arms and between arms of quadrivalents (interference)}

Observed distributions of RNs within arms of each quadrivalent for all but translocation $2-10 \mathrm{~b}$ are given in Table 2. Translocation $2-10 \mathrm{~b}$ was not included in this comparison because heterozygotes usually synapse as three-armed quadrivalents (Fig. 4) that are not readily compared with the four-armed quadrivalents characteristic of heterozygotes for the other four translocations studied.

To test for interference, observed distributions were compared with random distributions that were generated with the equation for a multinomial distribution (Devore, 1987).

$P=\left(m ! / n_{1} ! n_{2} ! n_{3} ! n_{4} !\right)(1 / 4)^{m}$,

where $P$ is the probability of a particular distribution of RNs between the arms of a quadrivalent when a certain total number of RNs $(m)$ is observed. $n_{1}-n_{4}$

Table 2 A comparison of random distribution of RNs to the observed distribution of RNs on different arms of quadrivalents for translocations 1-3,1-12,2-10a and 9-12. The expected percentage is the fraction of quadrivalents with a certain number $(\#)$ of RNs having a particular distribution based on a multinomial distribution (see text)*

\begin{tabular}{|c|c|c|c|c|c|c|c|c|c|c|c|c|c|c|c|c|c|c|c|c|c|c|c|c|c|c|}
\hline \multicolumn{2}{|c|}{$\begin{array}{l}\text { \# of RNs per } \\
\text { quadrivalent }\end{array}$} & 2 & & 3 & & & 4 & & & & & 5 & & & & & & 6 & & & & & & & & \\
\hline \multicolumn{27}{|c|}{$\begin{array}{l}\text { Possible RN } \\
\text { distribution }\end{array}$} \\
\hline arm 1 & & 2 & 1 & 3 & 2 & 1 & 4 & 3 & 2 & 2 & 1 & 5 & 4 & 3 & 3 & 2 & 2 & 6 & & & & & 3 & 3 & 2 & 2 \\
\hline $\operatorname{arm} 2$ & & 0 & 1 & 0 & 1 & 1 & 0 & 1 & 2 & 1 & 1 & 0 & 1 & 2 & 1 & 2 & 1 & 0 & 1 & 2 & . & 3 & 1 & 2 & 2 & 2 \\
\hline arm 3 & & 0 & 0 & 0 & 0 & 1 & 0 & 0 & 0 & 1 & 1 & 0 & 0 & 0 & 1 & 1 & 1 & 0 & 0 & 0 & 1 & 0 & 1 & 1 & 2 & 1 \\
\hline $\operatorname{arm} 4$ & & 0 & 0 & 0 & 0 & 0 & 0 & 0 & 0 & 0 & 1 & 0 & 0 & 0 & 0 & 0 & 1 & 0 & 0 & 0 & 0 & 0 & 1 & 0 & 0 & 1 \\
\hline Expected & & 25 & 75 & 6 & 56 & 38 & 2 & 19 & 14 & 56 & 9 & 0.3 & 6 & 12 & 23 & 35 & 23 & 0.1 & 2 & 4 & 9 & 3 & 12 & 35 & 9 & 26 \\
\hline \multicolumn{27}{|l|}{ Observed } \\
\hline \multirow[t]{2}{*}{$1-3$} & obs \# & 2 & 6 & 0 & 1 & 3 & 0 & 0 & 0 & 3 & 0 & 0 & 0 & 0 & 0 & 3 & 1 & 0 & 0 & 0 & 0 & 0 & 1 & 0 & 0 & 0 \\
\hline & obs $\%$ & 25 & 75 & 0 & 25 & 75 & 0 & 0 & 0 & 100 & 0 & 0 & 0 & 0 & 0 & 75 & 25 & 0 & 0 & 0 & 0 & 0 & 100 & 0 & 0 & 0 \\
\hline \multirow{2}{*}{$1-12$} & obs \# & 1 & 3 & 0 & 4 & 2 & 0 & 0 & 0 & 1 & 1 & 0 & 0 & 0 & 0 & 0 & 0 & 0 & 0 & 0 & 0 & 0 & 0 & 0 & 0 & 0 \\
\hline & obs $\%$ & 25 & 75 & 0 & 66 & 34 & 0 & 0 & 0 & 50 & 50 & 0 & 0 & 0 & 0 & 0 & 0 & 0 & 0 & 0 & 0 & 0 & 0 & 0 & 0 & 0 \\
\hline \multirow[t]{2}{*}{$2-10 \mathrm{~A}$} & obs \# & 0 & 2 & 0 & 3 & 2 & 0 & 0 & 0 & 2 & 0 & 0 & 0 & 0 & 1 & 1 & 0 & 0 & 0 & 0 & 0 & 0 & 0 & 0 & 0 & 3 \\
\hline & obs $\%$ & 0 & 100 & 0 & 60 & 40 & 0 & 0 & 0 & 100 & 0 & 0 & 0 & 0 & 50 & 50 & 0 & 0 & 0 & 0 & 0 & 0 & 0 & 0 & 0 & 100 \\
\hline \multirow[t]{2}{*}{$9-12$} & obs\# & 1 & 4 & 0 & 2 & 5 & 0 & 0 & 0 & 3 & 1 & 0 & 0 & 0 & 1 & 0 & 2 & 0 & 0 & 0 & 0 & 0 & 0 & 0 & 1 & 0 \\
\hline & obs $\%$ & 20 & 80 & 0 & 28 & 71 & 0 & 0 & 0 & 75 & 25 & 0 & 0 & 0 & 33 & 0 & 67 & 0 & 0 & 0 & 0 & 0 & 0 & 0 & 100 & 0 \\
\hline \multirow[t]{2}{*}{ Total } & obs \# & 4 & 15 & 0 & 10 & 12 & 0 & 0 & 0 & 9 & 2 & 0 & 0 & 0 & 2 & 4 & 3 & 0 & 0 & 0 & 0 & 0 & 1 & 0 & 1 & 3 \\
\hline & obs $\%$ & 21 & 79 & 0 & 41 & 59 & 0 & 0 & 0 & 82 & 18 & 0 & 0 & 0 & 22 & 45 & 33 & 0 & 0 & 0 & 0 & 0 & 20 & 0 & 20 & 60 \\
\hline
\end{tabular}

*Only quadrivalents having two or more RNs were considered in the analysis. 
represents a certain number of $\mathrm{RNs}$ that are observed in each arm. It was assumed that each of the four arms of the quadrivalent, regardless of its length, had an equal probability of having an $\mathrm{RN}$ [However, this assumption may represent a bias in the form of showing no interference because there is a positive correlation between the length of arms and the presence of RNs in non-translocated tomato chromosomes (Sherman et al., 1992)]. Arms were numbered randomly. For example, the probability of a quadrivalent with two RNs in arm 1, one $\mathrm{RN}$ in arm 2 and no RNs in arm 3 and arm 4 is [(3!/2!1!0!0!) $\left.(1 / 4)^{3}=\right] 3 / 64$. To determine the probability of having any combination of one arm with 2 RNs, one arm with 1 $\mathrm{RN}$, and two arms without RNs, the value of $P$ must be multiplied by all possible arrangements of the $\mathrm{RN}$ combination, in this case 12 , to give a probability of $[(3 / 64)(12)=] 36 / 64$ or 56 per cent. This calculation was repeated for all numbers of RNs observed.

A comparison of random distributions of RNs to the observed distributions by Chi-square analysis generally shows a general excess of quadrivalents having arms with one $\mathrm{RN}$ and a lack of quadrivalents having arms with two or more RNs (compare Expected percent to Total Observed percent [obs\%] in Table 2). The differences are significant based on a Chi-square test, for quadrivalents with 3,4 , and $5 \mathrm{RNs}(P=0.04,0.01$, and 0.05 respectively). These are the results anticipated if the presence of one nodule in an arm reduces (interferes with) the probability of another nodule in the same arm.

Table 3 Observed numbers of quadrivalents with RNs in only two arms, where the RNs are in either opposite or adjacent arms. The totals are compared to expected totals

- based on a random distribution

\begin{tabular}{llc}
\hline & \multicolumn{2}{l}{ RN position } \\
\cline { 2 - 3 } Translocation & Opposite & Adjacent \\
\hline $1-3$ & 2 & 5 \\
$1-12$ & 2 & 6 \\
$2-10 \mathrm{a}$ & 3 & 1 \\
$2-10 \mathrm{~b}^{*}$ & 4 & 6 \\
$9-12$ & 2 & 4 \\
Total & 13 & 22 \\
Expected & 11.25 & 23.75 \\
\hline
\end{tabular}

*The expected values for 2-10b were calculated independently of the other translocations because the translocated chromosomes usually synapsed to form a threearmed quadrivalent. Therefore, the expected ratio of RNs in opposite arms, versus adjacent arms, is 1:1 rather than $1: 2$. The expected values for 2-10b were added to the expected values for the other translocations.

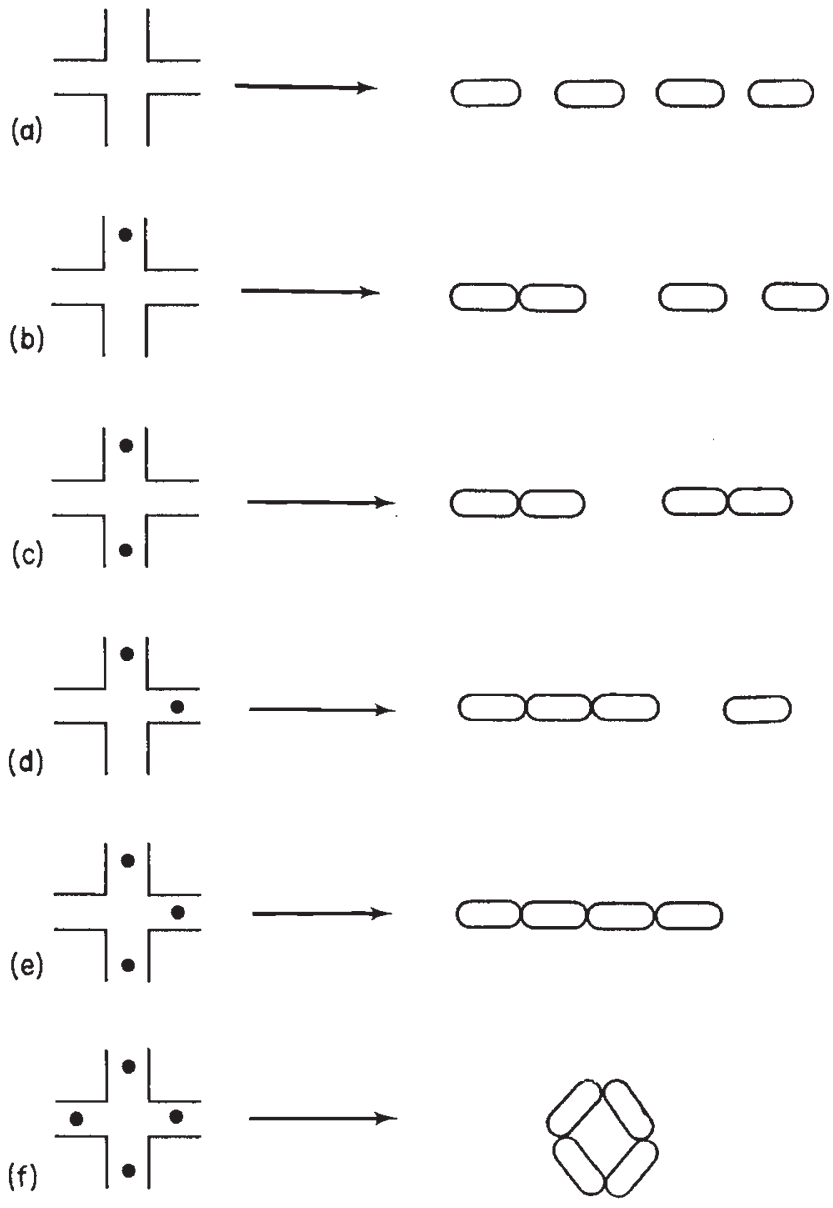

Fig. 6 Proposed relationship between RNs and chiasmata. To the left are pachytene quadrivalents in the form of a cross that results from homologous synapsis in a translocation heterozygote. Each continuous line represents one chromosome. Each black spot represents one or more RNs. Two chromosome arms that do not synapse are equivalent to synapsed arms lacking RNs. The ellipsoids to the right represent the resulting chromosome configurations at diakinesis if RNs lead to chiasmata. The sites where chromosomes touch represent one or more chiasmata. Since there is a large block of pericentric heterochromatin in each chromosome where very few crossovers occur, chiasmata link only the euchromatic ends of chromosomes. (a) A pachytene quadrivalent without RNs results in four univalents at diakinesis (see Fig. 7). (b) A pachytene quadrivalent with one or more RNs in only one arm results in a bivalent and two univalents at diakinesis (see Fig. 8). (c) A pachytene quadrivalent with one or more RNs in only two opposite arms results in two bivalents at diakinesis (see Fig. 9). (d) A pachytene quadrivalent with one or more RNs in only two adjacent arms results in a chain of three and one univalent at diakinesis (see Fig. 10). (e) A pachytene quadrivalent with one or more RNs in only three arms results in a chain of four chromosomes at diakinesis (see Fig. 11). (f) A pachytene quadrivalent with one or more RNs in all four arms results in a ring of four chromosomes at diakinesis (see Fig. 12). 
To determine whether $\mathrm{RN}$ interference is transmitted preferentially between opposite versus adjacent arms, quadrivalents with RNs in two arms only were examined to determine the proportion having RNs in opposite versus adjacent arms (Table 3, Fig. 6 c,d). If interference is not transmitted between arms, or transmitted equally, RNs should be found in adjacent arms twice as often as in opposite arms simply because there are twice as many arms adjacent to the first $\mathrm{RN}$, and only one arm opposite. This is what we observe (Table 3 ). However there is a potential bias in this analysis in that all arms are not equal in length or content of euchromatin. This could mean that certain arms are more likely to have an $\mathrm{RN}$ than others. In examining Fig. 13, it is clear that in at least three (translocation 1-3, $2-10 \mathrm{a}$, and $2-10 \mathrm{~b}$ ) of the tetravalents two of the opposite arms are significantly longer and have more euchromatin than the arms adjacent to them. This could result in a tendency toward more $\mathrm{RNs}$ in opposite compared with adjacent arms. In spite of this, only translocation $2-10$ a illustrated this tendency while all of the other translocations show a bias in favour of adjacent arms. Although the samples for each translocation are small, it seems clear that there is no overall tendency for RNs to be located in opposite arms. Indeed, when the data are combined and analysed by a Chi-square test, the differences between observed and expected location of RNs (based on random distributions) are not significant $(P=0.80)$. Thus it seems unlikely that interference is transmitted between arms.

\section{The relationship between patterns of synapsis and distribution of RNs}

To determine if there is a correlation between synapsis and the presence of RNs, we compared the number of cells from heterozygotes in which the translocated chromosomes synapsed as four-armed quadrivalents (or three synapsed arms in the case of translocation
$2-10 \mathrm{~b})$ to the number of cells in which quadrivalents had one or more RNs in each of four arms (or each of three synapsed arms for translocation 2-10b) (Table 4). Based on Fisher's exact test for $2 \times 2$ tables, the correlation between synapsis and the presence of RNs is not significant for any of the five translocation heterozygotes $(P<0.0014$ for each translocation).

\section{The relationship between patterns of synapsis and distribution of chiasmata}

To determine if there is a correlation between synapsis and the presence of chiasmata, we compared the number of cells from heterozygotes in which the translocated chromosomes synapsed as four-armed quadrivalents (or three synapsed arms in the case of translocation 2-10b) to the number of cells in which translocated chromosomes associated by chiasmata as a ring of four at diakinesis (or a chain of four for translocation 2-10b; Fig. 13). Based on a Fisher's exact test for $2 \times 2$ tables, the correlation between synapsis and chiasmata is not significant for any of the five translocation heterozygotes $(P<0.0001$ in all cases).

\section{The relationship between distribution of RNs and chiasmata}

If there is a $1: 1$ correlation between the presence of an $\mathrm{RN}$ in mid- to late pachytene and the occurrence of a chiasma at diakinesis, then in translocation heterozygotes a total lack of RNs in the translocated chromosomes will result in four univalents at diakinesis (Figs 6a and 7). One or more RNs in only one arm will result in a bivalent and two univalents at diakinesis (Figs 6b and 8). One or more RNs in two opposite arms only will result in two bivalents (Figs 6c and 9). One or more RNs in only two adjacent arms will result in a chain of three and one univalent chromosomes at diakinesis

Table 4 A comparison of (1) the number of quadrivalents with four arms synapsed to (2) the number of quadrivalents having a minimum of one RN in each of the four arms, and to (3) the number of diakinesis structures with a minimum of one chiasmata in each of four arms for each translocation. Values are expressed as a percent and the actual numbers are given in parentheses.

\begin{tabular}{llll}
\hline & $\begin{array}{l}\text { Pachytene quadrivalents with } \\
\text { 4 synapsed arms (3 synapsed } \\
\text { arms for 2-10b) }\end{array}$ & $\begin{array}{l}\text { Pachytene quadrivalents with } \\
\text { one or more RNs in each of } 4 \\
\text { synapsed arms }(3 \text { synapsed } \\
\text { arms for 2-10b) }\end{array}$ & $\begin{array}{l}\text { Diakinesis quadrivalents with } \\
\text { one or more chiasmata in } \\
\text { each of } 4 \text { arms }(3 \text { arms for } \\
2-10 \mathrm{~b})\end{array}$ \\
\hline $1-3$ & $80.7 \%(21 / 26)$ & $3.8 \%(2 / 26)$ & $10.0 \%(5 / 50)$ \\
$1-12$ & $85.7 \%(18 / 21)$ & $9.5 \%(2 / 21)$ & $11.5 \%(6 / 52)$ \\
$2-10 \mathrm{a}$ & $85.0 \%(17 / 20)$ & $20.0 \%(4 / 20)$ & $12.2 \%(6 / 49)$ \\
$2-10 \mathrm{~b}$ & $92.0 \%(23 / 25)$ & $32.0 \%(8 / 25)$ & $40.0 \%(20 / 50)$ \\
$9-12$ & $88.4 \%(23 / 26)$ & $11.5 \%(3 / 26)$ & $16.0 \%(8 / 50)$ \\
\hline
\end{tabular}



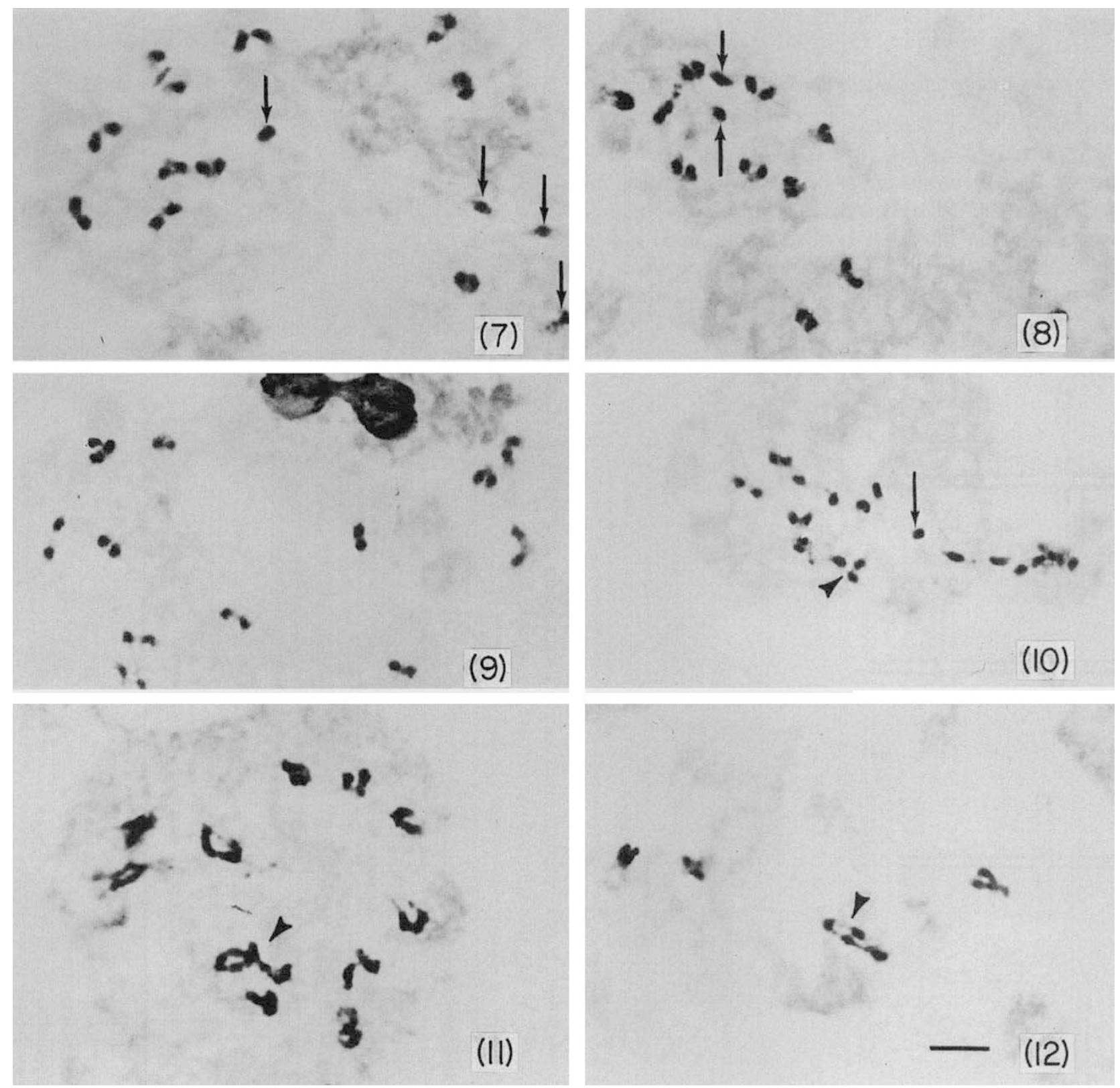

Fig. 7-12 Photomicrographs of chiasma configurations observed at diakinesis/metaphase I. 7 Ten bivalents and four univalents (arrows). 8 Eleven bivalents and two univalents (arrows). 9 Twelve bivalents. 10 Ten bivalents, a chain of three (arrow head), and one univalent (arrow). 11 Ten bivalents and a chain of four chromosomes (arrowhead). 12 Ten bivalents and a ring of four chromosomes (arrowhead). Bar equals $10 \mu \mathrm{m}$.

(Figs 6d and 10). One or more RNs in three of four arms will result in a chain of four chromosomes at diakinesis (Figs $6 \mathrm{e}$ and 11 ). One or more RNs in all four arms of a quadrivalent will result in a ring of four chromosomes at diakinesis (Figs $6 \mathrm{f}$ and 12).

Table 5 shows predicted percentages of distinct diakinesis configurations (based on observed percentages of different $\mathrm{RN}$ patterns) compared with the observed percentages of diakinesis configurations. Sets in which translocated chromosomes synapsed as bivalents were predicted to form bivalents at diakinesis since all bivalents had at least one $\mathrm{RN}$ at pachytene. When Chi-square tests were used to compare predicted and observed frequencies of diakinesis configurations for each translocation heterozygote, no significant differences were observed. The $P$ values are $0.47,0.83,0.29,0.18$ and 0.758 for translocations $2-10$ a, $2-10$ b, $9-12,1-3$, and $1-12$, respectively. Chisquare analyses could not consider groups where values of less than five existed, i.e. ring of four and four univalents (Table 5). 


\section{Discussion}

\section{Locating trans/ocation breakpoints}

In rye translocation heterozygotes, De Jong et al. (1989) reported that 0.01 to 13.76 per cent of the lengths of lateral elements are asynapsed, with increasing synapsis as the cells progressed from early to late pachytene. Borodin et al. (1991) observed up to 51 per cent asynapsis at late pachytene in reciprocal translocations of mice. They suggested that asynapsis could be
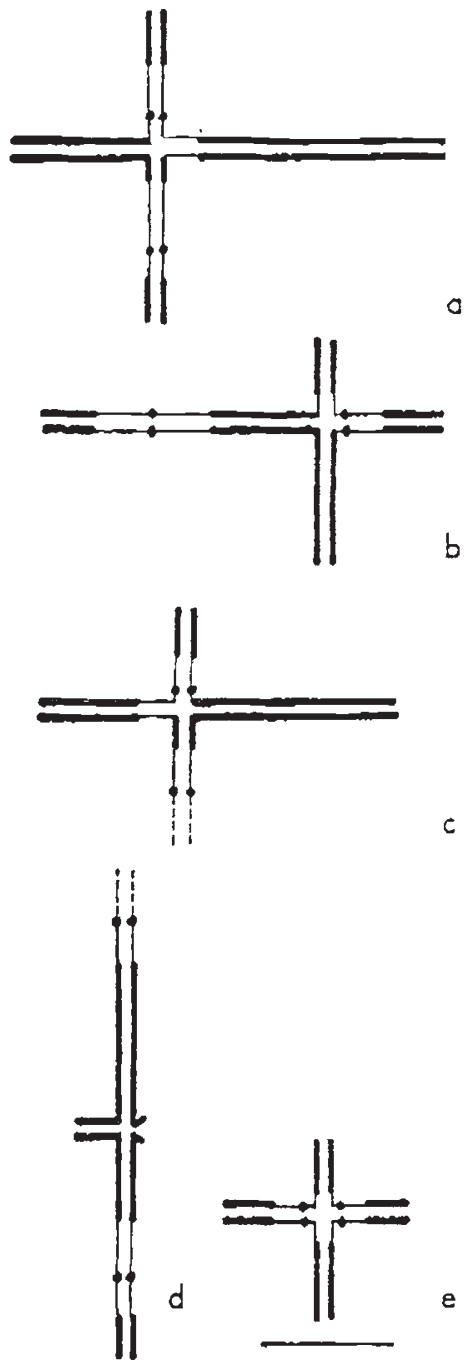

Fig. 13 Diagram illustrating the most common patterns of pachytene synapsis for five tomato translocation heterozygotes. (a) Translocation 1-3. (b) Translocation 1-12. (c) Translocation 1-10a. (d) Translocation 2-10b. (e) Translocation 9-12. SC lengths are based on a tomato idiogram by Sherman et al. (1992). Thick lines represent SC in euchromatin, thin lines represent SC in heterochromatin. Solid circles in heterochromatin represent centromeres. Bar equals $10 \mu \mathrm{m}$.
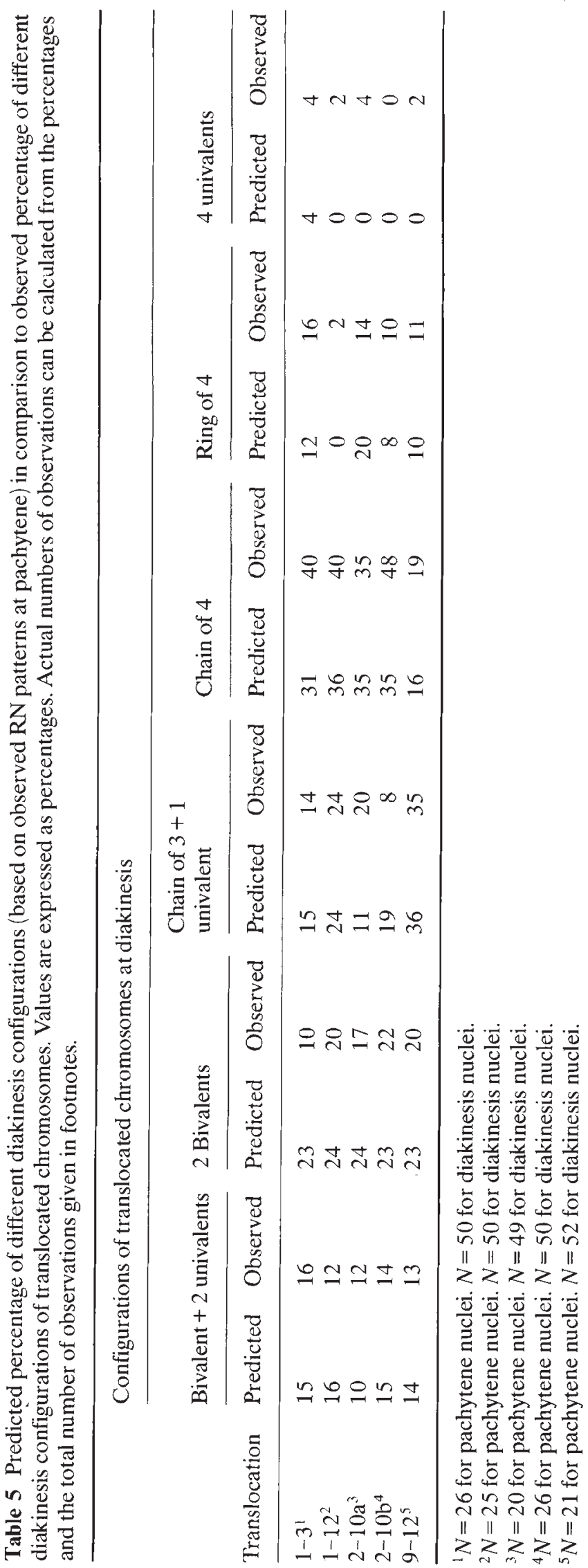
aggravated by structural heterozygosity and/or the spreading technique. Ashley \& Cacheiro (1990) found that positions of the breakpoints relative to G-bands in mouse $\mathrm{X}$-autosome translocations influenced the amount of asynapsis. In heterozygotes for the tomato translocations that we investigated, synapsis is usually in the form of a four-armed cross. Within each quadrivalent at the site of partner exchanges there is an area of asynapsis ranging from 10.5 to 16.4 per cent of the total length. While G-banding has not been reported in tomato, we did not find a difference in the total percentage of synapsis between translocations having breakpoints in euchromatin vs. heterochromatin.

In mouse translocation heterozygotes Moses et al. (1977) estimated the most probable location of breaks by averaging the middle points of asynapsed segments in quadrivalents. We used this method to locate translocation breaks in tomato chromosomes as well. Gill $e t$ al. (1980) examined squash preparations of heterozygotes for some of the same translocations (1-3, $2-10 \mathrm{a}, 2-10 \mathrm{~b}$, and $9-12$ ) and localized the breaks to euchromatin or heterochromatin of long or short arms. Our data confirm and refine the probable location of these breaks, and we have identified the breakpoints of translocation 1-12 for the first time. Additionally, there has been a question concerning whether translocation 1-3 involves chromosome 8 rather than 3 (Rick, 1985). We have shown that the translocation really involves chromosome 3 . Analysis of synaptic patterns in translocation heterozygotes is probably the most accurate cytological method for locating breakpoints in species that do not show G-banding.

\section{Effects of translocations on crossing over, chiasmata, and recombination nodules}

Heterozygosity for translocations has been reported both to increase and decrease crossing over and chiasmata in translocated chromosomes as compared with structurally normal bivalents. For example, in rye, Sybenga (1970) and Naranjo et al. (1989) found that translocation heterozygotes have an excess of chiasmata in quadrivalents as opposed to structurally normal chromosomes, while De Jong (1989) found a decrease in chiasmata in adjacent arms and an increase in chiasmata in opposite arms of quadrivalents. In mice Borodin et al. (1991) found an increase of chiasmata in heterozygotes for a translocation involving chromosome 17. Parker (1987) observed an increase in chiasma frequency only in regions spanning the partner exchange in Hypochoeris radicata interchange heterozygote. McKim et al. (1988) found severe and extensive recombination suppression near the breakpoints in translocation heterozygotes of Caenorhabditis elegans.
Dobzhansky (1931) and Roberts (1969) found that simple reciprocal translocations in Drosophila melanogaster are crossover suppressors, although sensitivity differed between chromosome arms. Lucchesi (1976) found that there are three effects of translocations on breakpoints based on distance of the breaks from centromeres. If breakpoints were far from centromeres, recombination was increased. If breakpoints were close to centromeres, recombination was decreased. Intermediate distances from the centromere to the breakpoint caused no change in recombination rates.

RNs show similarly mixed responses to translocations. In studying heterozygotes for Z-autosome translocations in Gallus domesticus, Solari et al. (1988) found no change in the position or frequency of strictly terminal RNs compared to normal bivalents involving the same chromosomes. Gillies (1979) also observed no significant difference in the number of RNs in normal bivalents versus translocated chromosomes in Neurospora crassa. However, in tomato we observed significant decreases in numbers of RNs/SC in five of six translocated chromosomes, i.e. all but chromosome 2. Here there are at least four possible explanations that are not mutually exclusive: (i) tomato quadrivalents average 85 per cent synapsis, thus eliminating 15 per cent of the available length for association with RNs. For chromosomes 9, 12 (translocation 9-12), and 10 (translocation 2-10b) increasing the number of RNs by 15 per cent results in a statistically insignificant difference between translocated and normal diploid chromosomes; (ii) based on observations of non-homologous synapsis in tomato trisomics (Sherman, unpublished observations), RNs do not associate with non-homologously synapsed segments of chromosomes. To the extent that there is some non-homologous synapsis in quadrivalents and bivalents, some undetermined length of SC is eliminated from associating with RNs; (iii) an RN in one arm of a quadrivalent can exert interference (see below) over four chromosomes in contrast to having its influence limited to two chromosomes in a bivalent; (iv) synapsis as a quadrivalent somehow generally interferes with crossing over. In support of the last explanation, whole quadrivalents may lack RNs (Table 5, Fig. 6a,b,d), whereas normal bivalents are invariably associated with at least one RN.

\section{Distribution of RNs within and between arms of quadrivalents (interference)}

Interference was first recognized in Drosophila as a reduction in the number of double crossovers compared with the number expected on the basis of random placement (Muller, 1916). Cytologically, inter- 
ference is defined as over dispersion of chiasmata compared with random placement (Haldane, 1931), i.e. chiasmata tend to be spaced apart along bivalents (Fox, 1973; Laurie \& Jones, 1981).

If RNs are indicative of crossover events, then RNs should demonstrate interference as well. In pachytene bivalents of Sordaria, Zickler (1977) reported that the distribution of RNs was variable but not random, although RNs did not show interference. On the other hand, human RNs have been reported to be overdispersed as would be expected with interference (Holm \& Rasmussen, 1983). Similarly, in tomato we have found that translocation heterozygotes show interference within arms because there are more arms with one RN and fewer arms with two or more RNs than expected on the basis of random distribution, this in spite of some bias in favour of arms with more than one $\mathrm{RN}$ because no adjustment was made for varying arm lengths (see Results). If interference extends from one arm containing an $\mathrm{RN}$ to other arms, it is not preferentially transferred to adjacent arms (as might be expected if interference were transmitted by lateral elements) vs. opposite arms. We base this conclusion on observations of quadrivalents with RNs in only two arms where the RNs are found in adjacent arms twice as often as in opposite arms (Figs 6c,d; Table 3). Alternatively, this observation may mean that interference is not transmitted between arms at all.

\section{The relationship of patterns of synapsis to distributions of RNs and chiasmata}

Homologous synapsis is accepted as a prerequisite for the high levels of crossing over observed in meiosis (Moses, 1968; von Wettstein et al., 1984). In the complete translocation heterozygote Rhoeo spathacea, where only the ends of chromosomes are synapsed, there seems to be a 1:1 correlation between synapsis of the ends of chromosomes and formation of chiasmata (Stack \& Soulliere, 1984). This might be explained by assuming that asynapsis in proximal segments blocks crossover interference. However, this must not be the case in tomato translocation heterozygotes because synapsed arms are joined by asynapsed lateral elements, and yet synapsed arms often lack RNs and fail to form chiasmata. A rationalization, if not an mechanistic explanation, for the regularity of chiasma formation in synapsed arms of Rhoeo is that Rhoeo, in contrast to tomato, is a naturally occurring translocation heterozygote that has been naturally selected for one or more chiasmata in each arm to encourage alternate disjunction and fertility.

\section{Correlation of chiasmata and RNs}

If a $\mathrm{RN}$ in late pachytene indicates the site of a crossover and subsequently a chiasma, the number and location of RNs should closely approximate the number and location of chiasmata. Indeed, in several cases where chiasmata are localized, RNs also show the same pattern of localization (Allium fistulosum - Albini \& Jones, 1984; Chloealtis conspersa - Bernelot-Moens \& Moens, 1986; Neocurtella hexadactyla - Spyropoulos et al., 1989). However, when frequencies of RNs and chiasmata are compared in nineteen different species including protists, fungi, plants, insects, birds, and mammals, good agreement (defined as less than 20 per cent variation) was found in ten species while there was poor agreement (more than 20 per cent variation) in the other nine (see Table 1 in Stack et al., 1989). The latter cases could be due to ephemeral RNs, artefactual loss or RNs, and/or difficulty in counting RNs and chiasmata accurately.

We had previously shown that the numbers of RNs observed in tomato closely approximates the number of crossovers estimated from the genetic map (Stack \& Anderson, 1986a,b). In the current study on tomato, we were largely able to avoid difficulties associated with counting chiasmata by using reciprocal translocations of chromosomes at diakinesis/metaphase I that indicate the location of a minimum of one chiasmata. In addition, based on the linkage map, most arms connected at diakinesis should have only one or two chiasmata. With these advantages, we were able to demonstrate that the predicted frequencies of chiasma distributions, based on the observed frequencies of RN distributions, are statistically indistinguishable from the observed frequencies of chiasma distributions for all five translocation heterozygotes investigated. This correlation and the observation that RNs show interference within arms strongly support a one RN: one crossover: one chiasma relationship in tomato.

\section{Acknowledgements}

We thank Dr Charles Rick of the Department of Vegetable Crops at the University of California at Davis for supplying translocation homozygote seeds; Lorrie Anderson and Dan Peterson for reading the manuscript; and Mark Anderson for writing the computer program for measuring SCs. The work was supported in part by grants DCB-8718170 and DCB8918613 from the National Science Foundation and grant 1878-670 from Colorado Agricultural Experiment Station. The work was done as partial fulfilment of M. S. requirements for L. Herickhoff. 


\section{References}

ALbINI, S. AND JONES, G. 1984. Synaptonemal complex-associated centromeres and recombination nodules in plant meiocytes prepared by an improved surface-spreading technique. Exp. Cell Res., 155, 589-592.

ASHLEY, T. AND CACHEIRO, N. L. A. 1990. Correlation between meiotic behavior and breakpoints with respect to $\mathrm{G}$-bands in two X-4 mouse translocations: $T(X ; 4) 7 R 1$ and T(X;4)8R1. Cytogenet. Cell Genet., 53, 178-184.

ASHLEY, T. AND RUSSELL, L. B. 1986. A new type of non-homologous synapsis in $\mathrm{T}(\mathrm{X} ; 4)$ IRI translocation male mice. Cytogenet. Cell Genet., 43, 194-200.

BERNELOT-MOENS, C. AND MOENS, P. 1986. Recombination nodules and chiasma localization in two Orthoptera. Chromosoma, 93, 220-226.

BORODIN, P., GORLOV, I., AGULNIK, A., AGULNIK, S. AND RUVINSKY, A. 1991. Chromosome pairing and recombination in mice heterozygous for different translocations in chromosome 16 and 17. Chromosoma, 101, 252-258.

DE JONG, J., VAN EDEN, J. AND SYBENGA, J. 1989. Synaptonemal complex formation and metaphase I configuration patterns in a translocation heterozygote of rye (Secale cereale). Genome, 32, 72-81.

DEvore, J. L. 1987. Probability and Statistics for Engineering and the Sciences. Brooks/Cole, Monterey.

DOBZHANSKY, TH. 1931. The decrease of crossing-over observed in translocations, and its probable explanation. Am. Nat., 698, 214-232.

FOX, D. P. 1973. The control of chiasma distribution in the locust. Schistocerca gregaria (Forskal). Chromosoma, 43, 289-328.

GILL, B. S., BURNHAM, C. R., STRINGHAM, G. R., STOUT, J. T. AND WEINHEIMER, H. 1980. Cytogenetic analysis of chromosomal translocations in the tomato: preferential breakage in heterochromatin. Can. J. Genet. Cytol., 22, 333-341.

GILLIES, C. 1979. The relationship between synaptonemal complexes, recombination nodules and crossing over in Neurospora crassa bivalents and translocation quadrivalents. Genetics, 91, 1-17.

HALDANE, J. B. 1931. The cytological basis of genetical interference. Cytologia, 3, 54-64.

HOLM, P. AND RASMUSSEN, s. 1983. Human meiosis VI. Crossing over in human spermatocytes. Carlesberg Res. Commun., 48, 385-413.

LAURIE, D. A. AND JONES, G. H. 1981. Inter-individual variation in chiasma frequency and chiasma distribution in Chorthippus brunneus. Heredity, 47, 409-416.

LUCCHESI, J. C. 1976. Interchromosomal effects. In: Ashburner, M. and Novitski, E. (eds) Genetics and Biology of Drosophila, Academic Press, N.Y., pp. 315-329.

McKIM, K. S., HOWELL, A. AND ROSE, A. 1988. The effects of translocations on recombination frequency in Caenorhabditis elegans. Genetics, 120, 987-1001.

Moses, M. 1968. Synaptinemal complex. Ann. Rev. Genet., 2, 363-412.

MOSES, M. J., RUSSELL, L. B. AND CACHEIRo, N. L. A. 1977. Mouse chromosome translocations: Visualization and analysis by electron microscopy of the synaptonemal complex. Science, 196, 892-894.
MULler, H. J. 1916. The mechanism of crossing-over. Am. Nat., 50, 193-221.

NARANJo, T., ROCA, A. AND GoICOECHEA, P. 1989. Comparison between synaptonemal complexes at pachytene and chromosome association at metaphase I in heterozygotes for a 'nonreciprocal' translocation of rye. Genome, 32, 983-991.

PARKER, J. S. 1987. Increased chiasma frequency as a result of chromosome rearrangements. Heredity, 58, 87-94.

RICK, C. 1985. Pt. II: List of Miscellaneous stocks. Translocations. Tomato Genet. Coop. Rep., 35, 27.

ROBERTS, P. 1969. Screening for X-Ray induced cross-over suppressors in Drosophila melanogaster: Prevalence and effectiveness of translocations. Genetics, 63, 429-448.

SHERMAN, J. AND STACK, s. 1992. Two-dimensional spreads of synaptonemal complexes from solanaceous plants. V. Tomato (Lycopersicon esculentum) karyotype and idiogram. Genome, 35, 354-359.

SHERMAN, J. D., HERICKHOFF, L. A. AND STACK, s. 1992. Silver staining two types of meiotic nodules. Genome, 35, 907-915.

SOLARI, A. J., FECHHEIMER, N. S. AND BITGOOD, J. J. 1988. Pairing of $\mathrm{ZW}$ gonosomes and the localized recombination nodule in two Z-autosome translocations in Gallus domesticus. Cytogenet. Cell Genet., 48, 130-136.

SPYROPOULOS, B. D., WISE, B. AND MOENS, P. 1989. Localized recombination nodules and sex chromosome behavior in the male mole cricket, Neocurtilla hexadactyla. Genome, 32, 275-281.

STACK, S. AND ANDERSON, L. 1986a. Two-dimensional spreads of synaptonemal complexes from solanaceous plants. II. Synapsis in Lycopersicon esculentum (tomato). Am. J. Bot., 73, 264-281.

STACK, S. AND ANDERSON, L. 1986b. Two-dimensional spreads of synaptonemal complexes from solanaceous plants. III. Recombination nodules and crossing over in Lycopersicon esculentum (tomato). Chromosoma, 94, 253-258.

STACK, S., ANDERSON, L. AND SHERMAN, J. 1989. Chiasmata and recombination nodules in Lilium longiflorum. Genome, 32, 486-498.

STACK, S. M. AND SOULLIERE, D. 1984. Rhoeo spathacea I. The relationship between synapsis and chiasma formation. Chromosoma, 90, 72-83.

SYBENGA, J. 1970. Simultaneous negative and positive chiasma interference across the breakpoint in interchange heterozygotes. Genetica, 41, 209-230.

TANKSLEY, S. D. AND MUTSCHLER, M. A. 1990. Linkage map of the tomato (Lycopersicon esculentum) $(2 N=24)$. In: O'Brien, S. J. (ed) Genetic maps: Locus maps of complex genomes Book 6 Plants, Cold Spring Harbor Laboratory Press, Cold Spring Harbor, pp. 6.3-6.15.

VON WETTSTEIN, D., RASMUSSEN, S. AND HOLM, P. 1984. The synaptonemal complex in genetic segregation. Ann. Rev. Genet., 18, 331-413.

ZICKLER, D. 1977. Development of the synaptonemal complex and the 'Recombination Nodules' during meiotic prophase in the seven bivalents of the fungus Sordaria macrospora Auersw. Chromosoma, 61, 289-316. 